\title{
376.
}

\section{THÉORÈME RELATIF À L'ÉQUILIBRE DE QUATRE FORCES.}

[From the Comptes Rendus de l'Académie des Sciences de Paris, tom. LXI. (JuilletDécembre 1865), pp. 829-830.]

ON sait que si quatre forces qui agissent sur un corps solide se tiennent en équilibre, alors (théorème de M. Möbius) les droites suivant lesquelles ces forces agissent sont quatre génératrices d'un même syperboloïde: et de plus en représentant chaque force par une longueur proportionnelle sur la direction de cette force, alors (théorème de M. Chasles) le tetraèdre formé par deux quelconques des forces est égal au tetraèdre formé par les deux autres forces.

En cherchant les valeurs des quatre forces lesquelles en agissant selon quatre génératrices données d'un même hyperboloïde se tiennent en équilibre, j'ai réussi à trouver pour ces valeurs une expression assez remarquable qui comprend comme corollaire le théorème de $\mathrm{M}$. Chasles.

Je nomme moment de deux droites la distance perpendiculaire de ces droites multipliée par le sinus de leur inclinaison mutuelle. Cela étant, en considérant quatre droites 1, 2, 3, 4 génératrices d'un même hyperboloïde, je dénote par ces mêmes symboles 1, 2, 3, 4 les forces qui agissent selon ces quatre droites respectivement, et par 12 le moment des droites 1 et 2, et de même pour les autres combinaisons de deux droites.

Or je dis que les forces 1,2,3,4 qui se tiennent en équilibre ont les valeurs proportionnelles que voici, à savoir en prenant les radicaux avec des signes convenables:

$$
\begin{aligned}
& 1=\sqrt{23.34 \cdot 42} \\
& 2=\sqrt{34.41 \cdot 13} \\
& 3=\sqrt{41.12 .24} \\
& 4=\sqrt{12.23 .31}
\end{aligned}
$$


On déduit de là, en écrivant pour abréger

$$
\nabla=\sqrt{23.31 \cdot 12 \cdot 14 \cdot 24 \cdot 34},
$$

les équations

$$
\begin{aligned}
& \nabla \sqrt{23.14}=2.3 \cdot 23=1.4 .14, \\
& \nabla \sqrt{31.24}=3 \cdot 1 \cdot 31=2.4 \cdot 24, \\
& \nabla \sqrt{12.34}=1 \cdot 2 \cdot 12=3.4 \quad 34,
\end{aligned}
$$

où par exemple l'équation $1.2 .12=3.4 .34$ exprime que le produit des forces 1 et 2 par le moment 12 des droites suivant lesquelles ces forces agissent est égal au produit des forces 3 et 4 par le moment 34 des droites selon lesquelles ces forces agissent.

J'ajoute que l'on a, en prenant les radicaux avec les signes convenables,

$$
\sqrt{23.14}+\sqrt{31 \cdot 24}+\sqrt{12 \cdot 34}=0,
$$

équation qui subsiste non seulement pour quatre génératrices quelconques d'un même hyperboloïde, mais pour quatre droites liées par une relation géométrique plus générale, à savoir, pour quatre droites telles que les deux droites qui rencontrent ces quatre droites se réduisent à une seule droite: ou (ce qui est la même chose) telles que chacune des quatre droites touche l'hyperboloïde qui passe par les trois autres droites. 